\title{
Multi-term fractional differential equations in a nonreflexive Banach space
}

\author{
Ravi P Agarwal ${ }^{1,2^{*}}$, Vasile Lupulescu ${ }^{3}$, Donal O'Regan ${ }^{4}$ and Ghaus ur Rahman ${ }^{5}$
}

${ }^{\text {*Correspondence: }}$

agarwal@tamuk.edu

1 Department of Mathematics, Texas A\&M University-Kingsville, Kingsville, TX, USA

${ }^{2}$ Department of Mathematics, King Abdulaziz University, P.O. Box 80203 Jeddah, 21859, Saudi Arabia Full list of author information is available at the end of the article

\section{严 Springer}

\begin{abstract}
In this paper we establish an existence result for the multi-term fractional differential equation

$$
\left(D^{\alpha_{m}}-\sum_{i=1}^{m-1} a_{i} D^{\alpha_{i}}\right) u(t)=f(t, u(t)) \quad \text { for } t \in[0,1], \quad u(0)=0
$$

where $D_{p}^{\alpha_{m}} y(\cdot)$ and $D_{p}^{\alpha_{i}} y(\cdot)$ are fractional pseudo-derivatives of a weakly absolutely continuous and pseudo-differentiable function $u(\cdot): T \rightarrow E$ of order $\alpha_{m}$ and $\alpha_{i}$, $i=1,2, \ldots, m-1$, respectively, the function $f(t, \cdot): T \times E \rightarrow E$ is weakly-weakly sequentially continuous for every $t \in T$ and $f(\cdot, y(\cdot))$ is Pettis integrable for every weakly absolutely continuous function $y(\cdot): T \rightarrow E, T$ is a bounded interval of real numbers and $E$ is a nonreflexive Banach space, $0<\alpha_{1}<\alpha_{2}<\cdots<\alpha_{m}<1$ and $a_{1}, a_{2}, \ldots, a_{m-1}$ are real numbers such that $a:=\sum_{i=1}^{m-1} \frac{\left|a_{i}\right|}{\Gamma\left(\alpha_{m}-\alpha_{i}+1\right)}<1$.
\end{abstract}

Keywords: weak measure of noncompactness; nonreflexive Banach spaces; Pettis integral; multi-term fractional differential equation; fractional pseudo-derivative

\section{Introduction}

The mathematical field that deals with derivatives of any real order is called fractional calculus. Fractional calculus has been successfully applied in various applied areas like computational biology, computational fluid dynamics and economics etc. [1].

In certain situations, we need to solve fractional differential equations containing more than one differential operator, and this type of fractional differential equation is called a multi-term fractional differential equation. Multi-term fractional differential equations have numerous applications in physical sciences and other branches of science [2]. The existence of solutions of multi-term fractional differential equations was studied by many authors [3-8]. The main tool used in [3-5] is the Krasnoselskii's fixed point theorem on a cone, while the main tool used in [7] is the technique associated with the measure of noncompactness and fixed point theorem. In [7], the author established the existence of a monotonic solution for a multi-term fractional differential equation in Banach spaces, using the Riemann-Liouville fractional derivative and in that paper no compactness condition is assumed on the nonlinearity of the function $f$.

When $\alpha_{m}=1$ and $a_{1}=a_{2} \cdots a_{m-1}=0$, the existence of weak solutions to multi-term fractional differential equation (1) was discussed in [9-13]. In [6], the author studied the existence of a weak solution of Cauchy problem (1) in reflexive Banach spaces equipped

○2013 Agarwal et al.; licensee Springer. This is an Open Access article distributed under the terms of the Creative Commons Attribution License (http://creativecommons.org/licenses/by/2.0), which permits unrestricted use, distribution, and reproduction in any medium, provided the original work is properly cited. 
with the weak topology, and the author assumed a weak-weak continuity assumption on $f$. In [8], the author established the existence of a global monotonic solution for Cauchy problem (1), and the author assumed $f$ is Carathéodory with linear growth.

In this present article, we prove the existence of a solution of Cauchy problem (1) in nonreflexive Banach spaces equipped with the weak topology. In comparison to other results in the literature, we use more general assumptions so that the function $f$ is assumed to be weakly-weakly sequentially continuous and $f(\cdot, u(\cdot))$ is Pettis integrable for each weakly absolutely continuous function $u(\cdot): T \rightarrow E$.

\section{Preliminaries}

For convenience here we present some notations and the main properties for Pettis integrable, weakly-weakly continuous functions, and we state some properties of the measure of noncompactness. Also, we present definitions and preliminary facts of fractional calculus in abstract spaces. Let $E$ be a Banach space with the norm $\|\cdot\|$, and let $E^{*}$ be the topological dual of $E$. If $x^{*} \in E^{*}$, then its value on an element $x \in E$ will be denoted by $\left\langle x^{*}, x\right\rangle$. The space $E$ endowed with the weak topology $\sigma\left(E, E^{*}\right)$ will be denoted by $E_{w}$. Consider an interval $T=[0, b]$ of $\mathbb{R}$ endowed with the Lebesgue $\sigma$-algebra $\mathcal{L}(T)$ and the Lebesgue measure $\lambda$. We will denote by $L^{1}(T)$ the space of all measurable and Lebesgue integrable real functions defined on $T$, and by $L^{\infty}(T)$ the space of all measurable and essentially bounded real functions defined on $T$.

Definition 2.1 (a) A function $x(\cdot): T \rightarrow E$ is said to be strongly measurable on $T$ if there exists a sequence of simple functions $x_{n}(\cdot): T \rightarrow E$ such that $\lim _{n \rightarrow \infty} x_{n}(t)=x(t)$ for a.e. $t \in T$.

(b) A function $x(\cdot): T \rightarrow E$ is said to be weakly measurable (or scalarly measurable) on $T$ if for every $x^{*} \in E^{*}$, the real-valued function $t \mapsto\left\langle x^{*}, x(t)\right\rangle$ is Lebesgue measurable on $T$. It is well known that a weakly measurable and almost separable valued function $x(\cdot): T \rightarrow E$ is strongly measurable [14, Theorem 1.1].

Definition 2.2 (a) A function $x(\cdot): T \rightarrow E$ is said to be absolutely continuous on $T$ ( $A C$, for short) if for every $\varepsilon>0$, there exists $\delta>0$ such that

$$
\left\|\sum_{k=1}^{m}\left[x\left(b_{k}\right)-x\left(a_{k}\right)\right]\right\|<\varepsilon
$$

for every finite disjoint family $\left\{\left(a_{k}, b_{k}\right) ; 1 \leq k \leq m\right\}$ of subintervals of $T$ such that $\sum_{k=1}^{m}\left(b_{k}-\right.$ $\left.a_{k}\right)<\delta$. If the last inequality is replaced by $\sum_{k=1}^{m}\left\|x\left(b_{k}\right)-x\left(a_{k}\right)\right\|<\varepsilon$, then we say that $x(\cdot)$ is a strongly absolutely continuous ( $S A C$ ) function.

(b) A function $x(\cdot): T \rightarrow E$ is said to be weakly absolutely continuous (wAC) on $T$ if for every $x^{*} \in E^{*}$, the real-valued function $t \mapsto\left\langle x^{*}, x(t)\right\rangle$ is $A C$ on $T$.

Remark 2.1 Each $s A C$ function is an $A C$ function, and each $A C$ function is a $w A C$ function. If $E$ is a weakly sequentially complete space, then every $w A C$ function is an $A C$ function [15]. 
Definition 2.3 (a) A function $x(\cdot): T \rightarrow E$ is said to be strongly differentiable at $t_{0} \in T$ if there exists an element $x_{s}^{\prime}\left(t_{0}\right) \in E$ such that

$$
\lim _{h \rightarrow 0}\left\|\frac{x\left(t_{0}+h\right)-x\left(t_{0}\right)}{h}-x_{s}^{\prime}\left(t_{0}\right)\right\|=0
$$

The element $x_{s}^{\prime}\left(t_{0}\right)$ will also be denoted by $\frac{d_{s}}{d t} x\left(t_{0}\right)$ and it is called the strong derivative of $x(\cdot)$ at $t_{0} \in T$.

(b) A function $x(\cdot): T \rightarrow E$ is said to be weakly differentiable at $t_{0} \in T$ if there exists an element $x_{w}^{\prime}\left(t_{0}\right) \in E$ such that

$$
\lim _{h \rightarrow 0}\left\langle x^{*}, \frac{x\left(t_{0}+h\right)-x\left(t_{0}\right)}{h}\right\rangle=\left\langle x^{*}, x_{w}^{\prime}\left(t_{0}\right)\right\rangle
$$

for every $x^{*} \in E^{*}$. The element $x_{w}^{\prime}\left(t_{0}\right)$ will be also denoted by $\frac{d_{w}}{d t} x\left(t_{0}\right)$ and it is called the weak derivative of $x(\cdot)$ at $t_{0} \in T$.

Proposition 2.1 [16, Theorem 7.3.3] If $E$ is a weakly sequentially complete space and $x(\cdot): T \rightarrow E$ is a function such that for every $x^{*} \in E^{*}$, the real function $t \mapsto\left\langle x^{*}, x(t)\right\rangle$ is differentiable $T$, then $x(\cdot)$ is weakly differentiable on $T$.

Proposition 2.2 [14, Theorem 1.2] If $x(\cdot): T \rightarrow E$ is an a.e. weakly differentiable on $T$, then its weak derivative $x_{w}^{\prime}(\cdot)$ is strongly measurable on $T$.

Definition 2.4 A function $x(\cdot): T \rightarrow E$ is said to be pseudo-differentiable on $T$ to a function $y(\cdot): T \rightarrow E$ if for every $x^{*} \in E^{*}$, there exists a null set $N\left(x^{*}\right) \in \mathcal{L}(T)$ such that the real function $t \mapsto\left\langle x^{*}, x(t)\right\rangle$ is differentiable on $T \backslash N\left(x^{*}\right)$ and

$$
\frac{d}{d t}\left\langle x^{*}, x(t)\right\rangle=\left\langle x^{*}, y(t)\right\rangle, \quad t \in T \backslash N\left(x^{*}\right) .
$$

The function $y(\cdot)$ is called a pseudo-derivative of $x(\cdot)$ and it will be denoted by $x_{p}^{\prime}(\cdot)$ or by $\frac{d p}{d t} x(\cdot)$.

Definition 2.5 A weakly measurable function $x(\cdot): T \rightarrow E$ is said to be Pettis integrable on $T$ if

(a) $x(\cdot)$ is scalarly integrable; that is, for every $x^{*} \in E^{*}$, the real function $t \mapsto\left\langle x^{*}, x(t)\right\rangle$ is Lebesgue integrable on $T$;

(b) for every set $A \in \mathcal{L}(T)$, there exists an element $x_{A} \in E$ such that

$$
\left\langle x^{*}, x_{A}\right\rangle=\int_{A}\left\langle x^{*}, x(s)\right\rangle d s
$$

for every $x^{*} \in E^{*}$. The element $x_{A} \in E$ is called the Pettis integral on $A$ and it will be denoted by $\int_{A} x(s) d s$.

Remark 2.2 (a) It is known that if $x(\cdot): T \rightarrow E$ is Bochner integrable on $T$, then the function $y(\cdot): T \rightarrow E$, given by

$$
y(t)=(B) \int_{0}^{t} x(s) d s, \quad t \in T
$$


is $A C$ and a.e. differentiable on $T$, and $y_{s}^{\prime}(t)=x(t)$ for a.e. $t \in T$. Also, if a function $x(\cdot)$ : $T \rightarrow E$ is $A C$ and a.e. strongly differentiable on $T$, then $x_{s}^{\prime}(\cdot)$ is Bochner integrable on $T$ and

$$
x(t)=x(0)+(B) \int_{0}^{t} x_{s}^{\prime}(s) d s, \quad t \in T .
$$

(b) In the case of the Pettis integral, in [14] it was shown that if $x(\cdot): T \rightarrow E$ is an $A C$ and a.e. weakly differentiable on $T$, then $x_{w}^{\prime}(\cdot)$ is Pettis integrable on $T$ and

$$
x(t)=x(0)+\int_{0}^{t} x_{w}^{\prime}(s) d s, \quad t \in T .
$$

In 1994 Kadets [17] proved that there exists a strongly measurable and Pettis integrable function $x(\cdot): T \rightarrow E$ such that the indefinite Pettis integral

$$
y(t)=\int_{0}^{t} x(s) d s, \quad t \in T
$$

is not weakly differentiable on a set of positive Lebesgue measures (see also $[18,19]$ ).

Proposition 2.3 [14] Let $x(\cdot): T \rightarrow$ E be a weakly measurable function.

(a) If $x(\cdot)$ is Pettis integrable on $T$, then the indefinite Pettis integral (4) is AC on T and $x(\cdot)$ is a pseudo-derivative of $y(\cdot)$.

(b) If $y(\cdot): T \rightarrow E$ is an AC function on $T$ and it has a pseudo-derivative $x(\cdot)$ on $T$, then $x(\cdot)$ is Pettis integrable on $T$ and

$$
y(t)=y(0)+\int_{0}^{t} x(s) d s, \quad t \in T .
$$

It is known that the Pettis integrals of two strongly measurable functions $x(\cdot): T \rightarrow E$ and $y(\cdot): T \rightarrow E$ coincide over every Lebesgue measurable set in $T$ if and only if $x(\cdot)=$ $y(\cdot)$ a.e. on $T$ [14, Theorem 5.2]. Since a pseudo-derivative of the pseudo-differentiable function $x(\cdot): T \rightarrow E$ is not unique and two pseudo-derivatives of $x(\cdot)$ need not be a.e. equal, then the concept of weak equivalence plays an important role in the following.

Definition 2.6 Two weak measurable functions $x(\cdot): T \rightarrow E$ and $y(\cdot): T \rightarrow E$ are said to be weakly equivalent on $T$ if for every $x^{*} \in E^{*}$, we have that $\left\langle x^{*}, x(t)\right\rangle=\left\langle x^{*}, y(t)\right\rangle$ for a.e. $t \in T$.

Proposition 2.4 A weakly measurable function $x(\cdot): T \rightarrow E$ is Pettis integrable on $T$ and $\left\langle x^{*}, x(\cdot)\right\rangle \in L^{\infty}(T)$ for every $x^{*} \in E^{*}$ if and only if the function $t \mapsto \varphi(t) x(t)$ is Pettis integrable on $T$ for every $\varphi(\cdot) \in L^{1}(T)$.

Let us denote by $P^{\infty}(T, E)$ the space of all weakly measurable and Pettis integrable functions $x(\cdot): T \rightarrow E$ with the property that $\left\langle x^{*}, x(\cdot)\right\rangle \in L^{\infty}(T)$ for every $x^{*} \in E^{*}$. Since for each $t \in T$ the real-valued function $s \mapsto(t-s)^{\alpha-1}$ is Lebesgue integrable on $[0, t]$ for every $\alpha>0$ then, by Proposition 2.4, the fractional Pettis integral

$$
I^{\alpha} x(t):=\int_{0}^{t} \frac{(t-s)^{\alpha-1}}{\Gamma(\alpha)} x(s) d s, \quad t \in T,
$$


exists for every function $x(\cdot) \in P^{\infty}(T, E)$ as a function from $T$ into $E$. Moreover, we have that

$$
\left\langle x^{*}, I^{\alpha} x(t)\right\rangle=\int_{0}^{t} \frac{(t-s)^{\alpha-1}}{\Gamma(\alpha)}\left\langle x^{*}, x(s)\right\rangle d s, \quad t \in T
$$

for every $x^{*} \in E^{*}$, and the real function $t \mapsto\left\langle x^{*}, I^{\alpha} x(t)\right\rangle$ is continuous (in fact, bounded and uniformly continuous on $T$ if $T=\mathbb{R}$ ) on $T$ for every $x^{*} \in E^{*}$ [20, Proposition 1.3.2].

Proposition 2.5 If $f(\cdot, \cdot): T \times E \rightarrow E$ is a function such that $f(\cdot, y(\cdot)) \in P^{\infty}(T, E)$ for every wAC function $y(\cdot): T \rightarrow E$, then the function $f_{\alpha}(\cdot)$ given by

$$
f_{\alpha}(t)=\int_{0}^{t} \frac{(t-s)^{1-\alpha}}{\Gamma(\alpha)} f(s, y(s)) d s, \quad t \in T
$$

has the following properties:

(a) $f_{\alpha}(\cdot)$ is $w A C$ on $T$;

(b) $f(\cdot, y(\cdot))$ is a pseudo-derivative of $f_{\alpha}(\cdot)$;

(c) $f_{\alpha}(0)=0$.

If $E$ is a weakly sequentially complete space, then $w A C$ is replaced by $A C$.

In the following, consider $\alpha \in(0,1)$. If $y(\cdot): T \rightarrow E$ is a pseudo-differentiable function with a pseudo-derivative $x(\cdot) \in P^{\infty}(T, E)$ on $T$, then the following fractional Pettis integral

$$
I^{1-\alpha} x(t)=\int_{0}^{t} \frac{(t-s)^{-\alpha}}{\Gamma(1-\alpha)} x(s) d s
$$

exists on $T$. The fractional Pettis integral $I^{1-\alpha} x(\cdot)$ is called a fractional pseudo-derivative of $y(\cdot)$ on $T$ and it will be denoted by $D_{p}^{\alpha} y(\cdot)$; that is,

$$
D_{p}^{\alpha} x(t)=I^{1-\alpha} x(t), \quad t \in T
$$

If $y(\cdot): T \rightarrow E$ is an a.e. weakly differentiable function with the weak derivative $y_{w}^{\prime}(\cdot) \in$ $P^{\infty}(T, E)$ on $T$, then

$$
D_{w}^{\alpha} y(t):=I^{1-\alpha} y_{w}^{\prime}(t), \quad t \in T,
$$

is called the fractional weak derivative of $y(\cdot)$ on $T$.

The following results will be useful (see [21] and [22]).

Remark 2.3 [21] If $y(\cdot): T \rightarrow E$ is a pseudo-differentiable function with a pseudoderivative $x(\cdot) \in P^{\infty}(T, E)$, then (a) $I^{\alpha} D_{p}^{\alpha} y(t)=y(t)-y(0)$ on $T$; (b) $D_{p}^{\alpha} I^{\alpha} y(t)=y(t)$ on $T$.

Remark 2.4 [21] The fractional Pettis integral is a linear operator from $P^{\infty}(T, E)$ into $P^{\infty}(T, E)$. Moreover, if $x(\cdot) \in P^{\infty}(T, E)$, then for $\alpha>0, \beta>0$, we have

$$
I^{\alpha} I^{\beta} x(t)=I^{\alpha+\beta} x(t), \quad t \in T .
$$




\section{Fractional differential equations}

In this section we establish an existence result for the fractional differential equation

$$
\left(D^{\alpha_{m}}-\sum_{i=1}^{m-1} a_{i} D^{\alpha_{i}}\right) u(t)=f(t, u(t)) \quad \text { for } t \in[0,1], \quad u(0)=0,
$$

where $D_{p}^{\alpha_{m}} y(\cdot)$ and $D_{p}^{\alpha_{i}} y(\cdot)$ are fractional pseudo-derivatives of the function $u(\cdot): T \rightarrow E$ of order $\alpha_{m}$ and $\alpha_{i}, i=1,2, \ldots, m-1$, respectively and $f(\cdot, \cdot): T \times E \rightarrow E$ is a given function, $0<\alpha_{1}<\alpha_{2}<\cdots<\alpha_{m}<1$ and $a_{1}, a_{2}, \ldots, a_{m-1}$ are real numbers such that $a:=\sum_{i=1}^{m-1} \frac{\left|a_{i}\right|}{\Gamma\left(\alpha_{m}-\alpha_{i}+1\right)}<1$. Along with Cauchy problem (9), consider the integral equation

$$
u(t)=\sum_{i=1}^{m-1} \int_{0}^{t} \frac{a_{i}(t-s)^{\left(\alpha_{m}-\alpha_{i}-1\right)}}{\Gamma\left(\alpha_{m}-\alpha_{i}\right)} u(s) d s+\int_{0}^{t} \frac{(t-s)^{\alpha_{m}-1}}{\Gamma\left(\alpha_{m}\right)} f(s, u(s)) d s, \quad t \in T
$$

where the integral is in the sense of Pettis.

Definition 3.1 A $w A C$ function (or an $A C$ function, if $E$ is a weakly sequentially complete space) $u(\cdot): T \rightarrow E$ is said to be a solution of (9) if

(i) $u(\cdot)$ has pseudo-derivative of order $\alpha_{i}, i=1,2, \ldots, n$,

(ii) the pseudo-derivative of $u(\cdot)$ of order $\alpha_{i}, i=1,2, \ldots, n$, belongs to $P^{\infty}(T, E)$,

(iii) $\left(D^{\alpha_{m}}-\sum_{i=1}^{m-1} a_{i} D^{\alpha_{i}}\right) u(t)=f(t, u(t))$ for all $t \in T$,

(iv) $u(0)=0$.

Lemma 3.1 Let $f(\cdot, \cdot): T \times E \rightarrow E$ be a function such that $f(\cdot, u(\cdot)) \in P^{\infty}(T, E)$ for every wAC function $u(\cdot): T \rightarrow E$. Then a wAC function $u(\cdot): T \rightarrow E$ is a solution of (9) if and only if it satisfies the integral equation (10).

Proof Indeed, if a $w A C$ function $u(\cdot): T \rightarrow E$ is a solution of (9), then from Remark 2.3(a) and Remark 2.4 it follows that $u(t)=\sum_{i=1}^{m-1} I^{\alpha_{m}-\alpha_{i}} a_{i} u(t)+I^{\alpha_{m}} f(t, u(t))$ on $T$; that is, $u(\cdot)$ satisfies the integral equation (10). Conversely, suppose that a $w A C$ function $u(\cdot)$ : $T \rightarrow E$ satisfies the integral equation (10). Since $f(\cdot, u(\cdot)) \in P^{\infty}(T, E)$, then from Proposition 2.5 it follows that the function $t \mapsto I^{\alpha_{m}} f(t, u(t))$ has a pseudo-derivative belonging to $P^{\infty}(T, E)$.Thus, using Remark 2.3(b), (10) and (6), we obtain that $\left(D^{\alpha_{m}}-\sum_{i=1}^{m-1} a_{i} D^{\alpha_{i}}\right) u(t)=$ $f(t, u(t))$ on $T$.

Let us denote by $P_{w k}(E)$ the set of all weakly compact subsets of $E$. The weak measure of noncompactness is the set function $\beta: P_{w k}(E) \rightarrow \mathbb{R}^{+}$defined by

$$
\beta(A)=\inf \left\{r>0 \text {; there exist } K \in P_{w k}(E) \text { such } A \subset K+r B_{1}\right\} \text {, }
$$

where $B_{1}$ is the closed unit ball in $E$. The properties of weak noncompactness measure are analogous to the properties of measure of noncompactness. If $A, B \in P_{w k}(E)$,

$\left(N_{1}\right) \quad A \subseteq B$ implies that $\beta(A) \leq \beta(B)$;

$\left(N_{2}\right) \quad \beta(A)=\beta\left(\mathrm{cl}_{w}(A)\right)$, where $\mathrm{cl}_{w}(A)$ denotes the weak closure of $A$;

$\left(N_{3}\right) \quad \beta(A)=0$ if and only if $\operatorname{cl}_{w}(A)$ is weakly compact;

$\left(N_{4}\right) \quad \beta(A \cup B)=\max \{\beta(A), \beta(B)\}$; 
$\left(N_{5}\right) \quad \beta(A)=\beta(\operatorname{conv}(A))$;

$\left(N_{6}\right) \quad \beta(A+B) \leq \beta(A)+\beta(B)$;

$\left(N_{7}\right) \quad \beta(x+A)=\beta(A)$, for all $x \in E$;

$\left(N_{8}\right) \quad \beta(\kappa A)=|\kappa| \beta(A)$, for all $\kappa \in \mathbb{R}$;

$\left(N_{9}\right) \quad \beta\left(\bigcup_{0 \leq r \leq r_{0}} r A\right)=r_{0} \beta(A)$;

$\left(N_{10}\right) \quad \beta(A) \leq 2 \operatorname{diam}(A)$.

Let $T=[0, a]$ and let $C(T, E)$ denote the space of all strong continuous functions $y(\cdot): T \rightarrow$ $E$, endowed with the supremum norm $\|y(\cdot)\|_{c}=\sup _{t \in T}\|y(t)\|$. Also, $C\left(T, E_{w}\right)$ denotes the space of all weakly continuous functions from $T$ into $E_{w}$ endowed with the topology of weak uniform convergence. It is known that (see $[23,24])$

$$
C(T, E)^{*}=M\left(T, E^{*}\right)
$$

where $M\left(T, E^{*}\right)$ is the space of all bounded regular vector measures from $\mathcal{B}(T)$ into $E^{*}$ which are of bounded variation. Here, $\mathcal{B}(T)$ denotes the $\sigma$-algebra of Borel measurable subsets of $T$.

Lemma 3.2 [25] Let $Y \subset C(T, E)$ be bounded and equicontinuous. Then

(i) the function $t \rightarrow \beta(Y(t))$ is continuous on $T$,

(ii) $\beta_{c}(Y)=\sup _{t \in T} \beta(Y(t))=\beta(Y(t))$,

where $\beta_{c}(\cdot)$ denotes the weak measure of noncompactness in $C(T, E)$ and $Y(t)=\{u(t) ; u \in$ $Y\}, t \in T$.

By a Kamke function we mean a function $g: \mathbb{R}_{+} \rightarrow \mathbb{R}_{+}$such that $g(\cdot)$ is continuous, nondecreasing with $g(0)=0$ and $u \equiv 0$ is the only solution of

$$
u(t) \leq \frac{1}{\Gamma(\alpha)} \int_{0}^{t}(t-s)^{\alpha-1} g(u(s)) d s, \quad u(0)=0 .
$$

We recall that a function $f(\cdot): E \rightarrow E$ is said to be sequentially continuous from $E_{w}$ into $E_{w}$ (or weakly-weakly sequentially continuous) if for every weakly convergent sequence $\left\{x_{n}\right\}_{n \geq 1} \subset E$, the sequence $\left\{f\left(x_{n}\right)\right\}_{n \geq 1}$ is weakly convergent in $E$.

Theorem 3.1 Let $r>0$. Let $f(\cdot, \cdot): T \times E \rightarrow E$ be a function such that:

(h1) $f(t, \cdot)$ is weakly-weakly sequentially continuous for every $t \in T$;

(h2) $f(\cdot, u(\cdot))$ is Pettis integrable for every $w A C$ function $u(\cdot): T \rightarrow E$;

(h3) $\|f(t, u)\| \leq M$ for all $(t, u) \in T \times B_{r}$, where $B_{r}=\left\{u \in E\right.$; $\left.\left\|u-u_{0}\right\| \leq r\right\}$;

(h4) for all $A \subseteq B_{r}$, we have

$$
\beta(f(T \times A)) \leq g(\beta(A)),
$$

where $g(\cdot)$ is a Kamke function. Then (9) admits a solution $u(\cdot)$ on an interval $\left[0, a_{0}\right]$ with

$$
a_{0}=\min \left\{1,\left[\frac{r(1-a) \Gamma\left(\alpha_{m}+1\right)}{M}\right]^{1 / \alpha_{m}}\right\}
$$


Proof Let $\widetilde{B}_{r}$ denote the set of all $w A C$ functions $u:\left[0, a_{0}\right] \rightarrow B_{r}$, and we consider the nonlinear operator $Q(\cdot)$ defined by

$$
(Q u)(t)=\sum_{i=1}^{m-1} \int_{0}^{t} \frac{a_{i}(t-s)^{\left(\alpha_{m}-\alpha_{i}-1\right)}}{\Gamma\left(\alpha_{m}-\alpha_{i}\right)} u(s) d s+\int_{0}^{t} \frac{(t-s)^{\alpha_{m}-1}}{\Gamma\left(\alpha_{m}\right)} f(s, u(s)) d s
$$

for all $t \in\left[0, a_{0}\right]$. Since the function $f(\cdot, u(t)) \in P^{\infty}(T, E)$ for every $u(\cdot) \in \widetilde{B}_{r}$, then by Proposition 2.5 it follows that the real function $t \mapsto(Q u)(t)$ is $w A C$ on $T$ for every $u(\cdot) \in \widetilde{B}_{r}$. Now, since

$$
\begin{aligned}
& \|(Q u)(t)\| \\
& =\left\|\sum_{i=1}^{m-1} \frac{a_{i}}{\Gamma\left(\alpha_{m}-\alpha_{i}\right)} \int_{0}^{t}(t-s)^{\alpha_{m}-\alpha_{i}-1} u(s) d s+\frac{1}{\Gamma\left(\alpha_{m}\right)} \int_{0}^{t}(t-s)^{\alpha_{m}-1} f(s, u(s)) d s\right\| \\
& \leq \sum_{i=1}^{m-1} \frac{r\left|a_{i}\right|}{\Gamma\left(\alpha_{m}-\alpha_{i}\right)} \int_{0}^{t}(t-s)^{\alpha_{m}-\alpha_{i}-1} d s+\frac{M}{\Gamma\left(\alpha_{m}\right)} \int_{0}^{t}(t-s)^{\alpha_{m}-1} d s \\
& \leq \sum_{i=1}^{m-1} \frac{r\left|a_{i}\right|}{\Gamma\left(\alpha_{m}-\alpha_{i}+1\right)}+\frac{M t^{\alpha_{m}}}{\Gamma\left(\alpha_{m}+1\right)} \leq r a+(1-a) r,
\end{aligned}
$$

we obtain that

$$
\|(Q u)(t)\| \leq r
$$

Thus $Q$ maps $\widetilde{B}_{r}$ into $\widetilde{B}_{r}$. Next, we show that $Q$ is weakly-weakly sequentially continuous. For this let $\left\{u_{n}(\cdot)\right\}_{n \geq 1}$ be a sequence in $C\left(\left[0, a_{0}\right], E\right)$ such that $u_{n}(\cdot) \stackrel{w}{\rightarrow} u(\cdot)$ as $n \rightarrow \infty$ (that is, $u_{n}(\cdot)$ converges weakly to $u(\cdot)$ in $\left.C\left(\left[0, a_{0}\right], E\right)\right)$. Since $\left(C\left(\left[0, a_{0}\right], E\right)^{*}\right)=M\left(\left[0, a_{0}\right], E^{*}\right)$, it follows that

$$
\left\langle m(\cdot), u_{n}(\cdot)-u(\cdot)\right\rangle \rightarrow 0 \quad \text { as } n \rightarrow \infty
$$

for all $m(\cdot) \in M\left(\left[0, a_{0}\right], E^{*}\right)$. Let $x^{*} \in E^{*}$ and $t \in\left[0, a_{0}\right]$. If we take $m(\cdot)=x^{*} \delta_{t}(\cdot)$, where $\delta_{t}$ is the Dirac measure concentrated in $\mathrm{t}$, then $m(\cdot) \in M\left(\left[0, a_{0}\right], E^{*}\right)$ and from (12) it follows that

$$
\left\langle x^{*}, u_{n}(t)-u(t)\right\rangle \rightarrow 0 \quad \text { as } n \rightarrow \infty
$$

Therefore, for each $t \in\left[0, a_{0}\right], u_{n}(t)$ converges weakly to $u(t)$ in $E$. Further, by (h1) it follows that $f\left(s, u_{n}(s)\right)$ converges weakly to $f(s, u(s))$ in $E$ for each $t \in\left[0, a_{0}\right]$. Hence, using Lemma 2.2 from [22], it follows that $I^{\alpha} u_{n}(\cdot)$ converges weakly to $I^{\alpha} u(\cdot)$ in $E$ for all $t \in\left[0, a_{0}\right]$, and also we have that $\left\{u_{n}\right\}$ converges to $u$ weakly. Then, by the Lebesgue dominated convergence theorem for Pettis integral (see [23]), we obtain

$$
\begin{aligned}
\lim _{n \rightarrow \infty}\left\langle m(\cdot), Q u_{n}(\cdot)-Q u(\cdot)\right\rangle= & \lim _{n \rightarrow \infty} \int_{0}^{a_{0}}\left[\sum_{i=1}^{m-1} \frac{a_{i}}{\Gamma\left(\alpha_{m}-\alpha_{i}\right)} \int_{0}^{t}(t-s)^{\alpha_{m}-\alpha_{i}-1} u_{n}(s) d s\right. \\
& +\frac{1}{\Gamma\left(\alpha_{m}\right)} \int_{0}^{t}(t-s)^{\alpha_{m}-1} f\left(s, u_{n}(s)\right) d s
\end{aligned}
$$




$$
\begin{aligned}
& -\sum_{i=1}^{m-1} \frac{a_{i}}{\Gamma\left(\alpha_{m}-\alpha_{i}\right)} \int_{0}^{t}(t-s)^{\alpha_{m}-\alpha_{i}-1} u(s) d s \\
& \left.-\frac{1}{\Gamma\left(\alpha_{m}\right)} \int_{0}^{t}(t-s)^{\alpha_{m}-1} f(s, u(s)) d s\right] d m(s), \\
\lim _{n \rightarrow \infty}\left\langle m(\cdot), Q u_{n}(\cdot)-Q u(\cdot)\right\rangle= & \lim _{n \rightarrow \infty} \int_{0}^{a_{0}}\left\{\sum_{i=1}^{m-1} \int_{0}^{t} \frac{(t-s)^{\alpha_{m}-\alpha_{i}-1} a_{i}\left[u_{n}(s)-u(s)\right]}{\Gamma\left(\alpha_{m}-\alpha_{i}\right)} d s\right. \\
& \left.+\frac{1}{\Gamma\left(\alpha_{m}\right)} \int_{0}^{t}(t-s)^{\alpha_{m}-1}\left[f\left(s, u_{n}(s)\right)-f(s, u(s))\right] d s\right\} d m(s) \\
= & \lim _{n \rightarrow \infty} \int_{0}^{a_{0}}\left[\frac{1}{\Gamma\left(\alpha_{m}\right)} \int_{0}^{t}(t-s)^{\alpha_{m}-1} f\left(s, u_{n}(s)\right) d s\right. \\
& \left.-\frac{1}{\Gamma\left(\alpha_{m}\right)} \int_{0}^{t}(t-s)^{\alpha_{m}-1} f(s, u(s)) d s\right] d m(s) \\
= & \lim _{n \rightarrow \infty} \int_{0}^{a_{0}}\left[I^{\alpha} u_{n}(t)-I^{\alpha} u(t)\right] d m(s)=0
\end{aligned}
$$

for all $m(\cdot) \in M\left(\left[0, a_{0}\right], E^{*}\right)$. Therefore, $Q$ is weakly-weakly continuous. Next, for any $n \geq 1$, we define the sequence $\left\{u_{n}(\cdot)\right\}_{n \geq 1}$ as follows:

$$
\begin{aligned}
& u_{n}(t)=0 \quad \text { if } 0 \leq t \leq \frac{a_{0}}{n} \\
& u_{n}(t)=\sum_{i=1}^{m-1} \int_{0}^{t} \frac{a_{i}(t-s)^{\left(\alpha_{m}-\alpha_{i}-1\right)}}{\Gamma\left(\alpha_{m}-\alpha_{i}\right)} u_{n}(t) d s+\int_{0}^{t} \frac{(t-s)^{\alpha_{m}-1}}{\Gamma\left(\alpha_{m}\right)} f\left(s, u_{n}(t)\right) d s \quad \text { if } \frac{a_{0}}{n} \leq t \leq a_{0} .
\end{aligned}
$$

Obviously, $u_{n} \in \widetilde{B}_{r}$ for all $n \geq 1$. Further, for all $n \geq 1$, if $t \in\left[0, a_{o} / n\right]$, then

$$
\begin{aligned}
\left\|u_{n}(t)-\left(Q u_{n}(t)\right)\right\|= & \left\|\left(Q u_{n}\right)(t)\right\| \\
= & \| \sum_{i=1}^{m-1} \frac{a_{i}}{\Gamma\left(\alpha_{m}-\alpha_{i}\right)} \int_{0}^{t}(t-s)^{\alpha_{m}-\alpha_{i}-1} u_{n}(s) d s \\
& +\frac{1}{\Gamma\left(\alpha_{m}\right)} \int_{0}^{t}(t-s)^{\alpha_{m}-1} f\left(s, u_{n}(s)\right) d s \| \\
\leq & \sum_{i=1}^{m-1} \frac{r\left|a_{i}\right| t^{\alpha_{m}-\alpha_{i}}}{\Gamma\left(\alpha_{m}-\alpha_{i}\right)\left(\alpha_{m}-\alpha_{i}\right)}+\frac{M t^{\alpha_{m}}}{\Gamma\left(\alpha_{m}\right)} \\
= & \sum_{i=1}^{m-1} \frac{r\left|a_{i}\right|\left(a_{0} / n\right)^{\alpha_{m}-\alpha_{i}}}{\Gamma\left(\alpha_{m}-\alpha_{i}+1\right)}+\frac{M\left(a_{0} / n\right)^{\alpha_{m}}}{\Gamma\left(\alpha_{m}+1\right)} .
\end{aligned}
$$

If $t \in\left[a_{0} / n, a_{0}\right]$, then

$$
\begin{aligned}
\left\|u_{n}(t)-\left(Q u_{n}\right)(t)\right\|= & \|\left(\sum_{i=1}^{m-1} \frac{a_{i}}{\Gamma\left(\alpha_{m}-\alpha_{i}\right)} \int_{0}^{t-a_{0} / n}(t-s)^{\alpha_{m}-\alpha_{i}-1} u_{n}(s) d s\right. \\
& +\frac{1}{\Gamma\left(\alpha_{m}\right)} \int_{0}^{t-a_{0} / n}(t-s)^{\alpha_{m}-1} f\left(s, u_{n}(s)\right) d s
\end{aligned}
$$




$$
\begin{aligned}
& -\sum_{i=1}^{m-1} \frac{a_{i}}{\Gamma\left(\alpha_{m}-\alpha_{i}\right)} \int_{0}^{t}(t-s)^{\alpha_{m}-\alpha_{i}-1} u_{n}(s) d s \\
& \left.-\frac{1}{\Gamma\left(\alpha_{m}\right)} \int_{0}^{t}(t-s)^{\alpha_{m}-1} f\left(s, u_{n}(s)\right) d s\right) \| \\
= & \| \sum_{i=1}^{m-1} \frac{a_{i}}{\Gamma\left(\alpha_{m}-\alpha_{i}\right)}\left[\int_{0}^{t}(t-s)^{\alpha_{m}-\alpha_{i}-1} u_{n}(s) d s\right. \\
& \left.-\int_{0}^{t-a_{0} / n}(t-s)^{\alpha_{m}-\alpha_{i}-1} u_{n}(s) d s\right] \\
& +\frac{1}{\Gamma\left(\alpha_{m}\right)}\left[\int_{0}^{t}(t-s)^{\alpha_{m}-1} f\left(s, u_{n}(s)\right) d s\right. \\
& \left.-\int_{0}^{t-a_{0} / n}(t-s)^{\alpha_{m}-1} f\left(s, u_{n}(s)\right) d s\right] \| \\
\leq & \sum_{i=1}^{m-1} \frac{r\left|a_{i}\right|\left(a_{0} / n\right)^{\alpha_{m}-\alpha_{i}}}{\Gamma\left(\alpha_{m}-\alpha_{i}+1\right)}+\frac{M\left(a_{0} / n\right)^{\alpha_{m}}}{\Gamma\left(\alpha_{m}+1\right)} .
\end{aligned}
$$

Thus we obtain that for all $t \in\left[0, a_{0}\right]$,

$$
\lim _{n \rightarrow \infty}\left\|u_{n}-Q u_{n}\right\|_{c}=0
$$

Let $V=\left\{u_{n}(\cdot) ; n \geq 1\right\}$ and $W=Q(V)=\left\{Q u_{n}(\cdot) ; n \geq 1\right\}$. If $s, t \in\left[0, a_{0} / n\right]$ and $s<t$, then

$$
\begin{aligned}
\left\|Q u_{n}(s)-Q u_{n}(t)\right\|= & \left\|\int_{0}^{t} \frac{(t-\tau)^{\alpha_{m}-1} f(\tau, 0)}{\Gamma\left(\alpha_{m}\right)} d \tau-\int_{0}^{s} \frac{(s-\tau)^{\alpha_{m}-1}}{\Gamma\left(\alpha_{m}\right)} f(\tau, 0) d \tau\right\| \\
= & \| \int_{0}^{s} \frac{(t-\tau)^{\alpha_{m}-1} f(\tau, 0)}{\Gamma\left(\alpha_{m}\right)} d \tau+\int_{s}^{t} \frac{(t-\tau)^{\alpha_{m}-1} f(\tau, 0)}{\Gamma\left(\alpha_{m}\right)} d \tau \\
& -\int_{0}^{s} \frac{(s-\tau)^{\alpha_{m}-1}}{\Gamma\left(\alpha_{m}\right)} f(\tau, 0) d \tau \| \\
\leq & \frac{M}{\Gamma\left(\alpha_{m}\right)} \int_{0}^{s}\left[(s-\tau)^{\alpha_{m}-1}-(t-\tau)^{\alpha_{m}-1}\right] d \tau \\
& +\frac{M}{\Gamma\left(\alpha_{m}\right)} \int_{s}^{t}(t-\tau)^{\alpha_{m}-1} d \tau \\
\leq & \frac{2 M}{\Gamma\left(\alpha_{m}+1\right)}\left[(t-s)^{\alpha_{m}}\right] .
\end{aligned}
$$

If $s, t \in\left[a_{0} / n, a_{0}\right]$ and $s<t$, then

$$
\begin{aligned}
\left\|Q u_{n}(t)-Q u_{n}(s)\right\|= & \| \sum_{i=1}^{m-1} \frac{a_{i}}{\Gamma\left(\alpha_{m}-\alpha_{i}\right)}\left[\int_{0}^{t}(t-\tau)^{\alpha_{m}-\alpha_{i}-1} u_{n}(\tau) d \tau\right. \\
& \left.-\int_{0}^{s}(s-\tau)^{\alpha_{m}-\alpha_{i}-1} u_{n}(\tau) d \tau\right] \\
& +\frac{1}{\Gamma\left(\alpha_{m}\right)}\left[\int_{0}^{t}(t-\tau)^{\alpha_{m}-1} f\left(\tau, u_{n}(\tau)\right) d \tau\right. \\
& \left.-\int_{0}^{s}(s-\tau)^{\alpha_{m}-1} f\left(\tau, u_{n}(\tau)\right) d \tau\right] \|
\end{aligned}
$$




$$
\begin{aligned}
\leq & \| \sum_{i=1}^{m-1} \frac{a_{i}}{\Gamma\left(\alpha_{m}-\alpha_{i}\right)}\left[\int_{0}^{s}\left[(s-\tau)^{\alpha_{m}-\alpha_{i}-1}-(t-\tau)^{\alpha_{m}-\alpha_{i}-1}\right] u_{n}(\tau) d \tau\right. \\
& \left.-\int_{s}^{t}(t-\tau)^{\alpha_{m}-\alpha_{i}-1} u_{n}(\tau) d \tau\right] \| \\
& +\| \frac{1}{\Gamma\left(\alpha_{m}\right)}\left[\int_{0}^{s}\left[(s-\tau)^{\alpha_{m}-1}-(t-\tau)^{\alpha_{m}-1}\right] f\left(\tau, u_{n}(\tau)\right) d \tau\right. \\
& \left.-\int_{s}^{t}(t-\tau)^{\alpha_{m}-1} f\left(\tau, u_{n}(\tau)\right) d \tau\right] \| \\
\leq & \sum_{i=1}^{m-1} \frac{r\left|a_{i}\right|}{\Gamma\left(\alpha_{m}-\alpha_{i}+1\right)}\left[\int_{0}^{s}\left[(s-\tau)^{\alpha_{m}-\alpha_{i}-1}-(t-\tau)^{\alpha_{m}-\alpha_{i}-1}\right] d \tau\right] \\
& +\int_{s}^{t}(t-\tau)^{\alpha_{m}-\alpha_{i}-1} d \tau \\
& +\frac{M}{\Gamma\left(\alpha_{m}\right)}\left[\int_{0}^{s}\left[(s-\tau)^{\alpha_{m}-1}-(t-\tau)^{\alpha_{m}-1}\right] d \tau\right. \\
& \left.+\int_{s}^{t}(t-\tau)^{\alpha_{m}-1} d \tau\right] \\
\leq & 2\left[\sum_{i=1}^{m-1} \frac{r\left(\alpha_{m}-\alpha_{i}+1\right)}{\Gamma\left(a_{i} \mid\right.} \frac{M}{\Gamma\left(\alpha_{m}+1\right)}\right](t-s)^{\alpha_{m}} \cdot
\end{aligned}
$$

If $s \in\left[0, a_{0} / n\right]$ and $t \in\left[a_{0} / n, a_{0}\right]$, then

$$
\begin{aligned}
& \left\|Q u_{n}(s)-Q u_{n}(t)\right\|=\| \int_{0}^{s} \frac{(s-\tau)^{\alpha_{m}-1}}{\Gamma\left(\alpha_{m}\right)} f(\tau, 0) d s-\sum_{i=1}^{m-1} \int_{0}^{t} \frac{a_{i}(t-\tau)^{\left(\alpha_{m}-\alpha_{i}-1\right)}}{\Gamma\left(\alpha_{m}-\alpha_{i}\right)} u_{n}(\tau) d \tau \\
& -\int_{0}^{t} \frac{(t-\tau)^{\alpha_{m}-1}}{\Gamma\left(\alpha_{m}\right)} f\left(\tau, u_{n}(\tau)\right) d s \| \\
& =\| \frac{1}{\Gamma\left(\alpha_{m}\right)} \int_{0}^{s}\left[(t-\tau)^{\alpha_{m}-1}-(s-\tau)^{\alpha_{m}-1}\right] f(\tau, 0) d \tau \\
& +\int_{s}^{t} \frac{(t-\tau)^{\alpha_{m}-1}}{\Gamma\left(\alpha_{m}\right)} f\left(\tau, u_{n}(\tau)\right) d s \\
& +\sum_{i=1}^{m-1} \int_{s}^{t} \frac{a_{i}(t-\tau)^{\left(\alpha_{m}-\alpha_{i}-1\right)}}{\Gamma\left(\alpha_{m}-\alpha_{i}\right)} u_{n}(\tau) d \tau \| \\
& \leq \frac{M}{\Gamma\left(\alpha_{m}\right)} \int_{0}^{s}\left[(t-\tau)^{\alpha_{m}-1}-(s-\tau)^{\alpha_{m}-1}\right] d \tau \\
& +\int_{s}^{t} \frac{M(t-\tau)^{\alpha_{m}-1}}{\Gamma\left(\alpha_{m}\right)} d \tau+\sum_{i=1}^{m-1} \int_{s}^{t} \frac{\left|a_{i}\right| r(t-\tau)^{\left(\alpha_{m}-\alpha_{i}-1\right)}}{\Gamma\left(\alpha_{m}-\alpha_{i}\right)} d \tau \\
& \leq \frac{M}{\Gamma\left(\alpha_{m}+1\right)}\left[s^{\alpha_{m}}+(t-s)^{\alpha_{m}}-t^{\alpha_{m}}+(t-s)^{\alpha_{m}}\right] \\
& +\frac{r \sum_{i=1}^{m-1}}{\Gamma\left(\alpha_{m}-\alpha_{i}+1\right)}(t-s)^{\alpha_{m}-\alpha_{i}} \\
& \leq\left[\frac{2 M}{\Gamma\left(\alpha_{m}+1\right)}+\frac{r \sum_{i=1}^{m-1}}{\Gamma\left(\alpha_{m}-\alpha_{i}+1\right)}\right](t-s)^{\alpha_{m}} \text {. }
\end{aligned}
$$


It follows from the above three cases that $W$ is equicontinuous, and by (13) we obtain that $V$ is also equicontinuous. By virtue of Lemma 3.2 and (13), we have that

$$
\begin{aligned}
\beta_{c}(V-W) & =\beta((I-Q)(V)) \\
& =\sup _{t \in\left[0, a_{0}\right]} \beta(I-Q)(V(t))=0 .
\end{aligned}
$$

Since for all $t \in\left[0, a_{0}\right]$ we have that $V(t) \subseteq V(t)-W(t)+W(t)$, it follows that

$$
\beta(V(t)) \leq \beta(W(t)) \text { for } t \in\left[0, a_{0}\right]
$$

On the other hand, for all $t \in\left[0, a_{0}\right]$, we have $W(t) \subseteq W(t)-V(t)+V(t)=V(t)-(I-$ $Q)(V(t))$, and so

$$
\beta(W(t)) \leq \beta(V(t)) \text { for } t \in\left[0, a_{0}\right]
$$

From (14) and (15), we infer that

$$
\beta(W(t))=\beta(V(t)) \quad \text { for } t \in\left[0, a_{0}\right] .
$$

Further, fix $t \in T_{0}, \varepsilon>0$ and choose $\eta>0$ such that $\eta<\left(\frac{\varepsilon \Gamma\left(\alpha_{m}+1\right)}{M+r \Gamma\left(\alpha_{m}+1\right)}\right)^{1 / \alpha_{m}}$. Then

$$
\begin{aligned}
& \left\|\sum_{i=1}^{m-1} \frac{a_{i}}{\Gamma\left(\alpha_{m}-\alpha_{i}\right)} \int_{t-\eta}^{t}(t-s)^{\alpha_{m}-\alpha_{i}-1} u(s) d s+\frac{1}{\Gamma\left(\alpha_{m}\right)} \int_{t-\eta}^{t}(t-s)^{\alpha_{m}-1} f(s, u(s)) d s\right\| \\
& \quad \leq \sum_{i=1}^{m-1} \frac{r\left|a_{i}\right| \eta^{\alpha_{m}-\alpha_{i}}}{\Gamma\left(\alpha_{m}-\alpha_{i}+1\right)}+\frac{M \eta^{\alpha_{m}}}{\Gamma\left(\alpha_{m}+1\right)} \\
& \quad \leq \sum_{i=1}^{m-1} \frac{r\left|a_{i}\right| \eta^{\alpha_{m}}}{\Gamma\left(\alpha_{m}-\alpha_{i}+1\right)}+\frac{M \eta^{\alpha_{m}}}{\Gamma\left(\alpha_{m}+1\right)} \\
& \quad \leq r \eta^{\alpha_{m}}+\frac{M \eta^{\alpha_{m}}}{\Gamma\left(\alpha_{m}+1\right)} \\
& \quad \leq \frac{M+r \Gamma\left(\alpha_{m}+1\right)}{\Gamma\left(\alpha_{m}+1\right)} \eta^{\alpha_{m}}<\varepsilon .
\end{aligned}
$$

Hence we conclude that

$$
\left\|\sum_{i=1}^{m-1} \frac{a_{i}}{\Gamma\left(\alpha_{m}-\alpha_{i}\right)} \int_{t-\eta}^{t}(t-s)^{\alpha_{m}-\alpha_{i}-1} V(s) d s+\frac{1}{\Gamma\left(\alpha_{m}\right)} \int_{t-\eta}^{t}(t-s)^{\alpha_{m}-1} f(s, u(s)) d s\right\|<\varepsilon .
$$

Using the property $\left(N_{10}\right)$ of noncompactness measure, we infer

$$
\beta\left(\sum_{i=1}^{m-1} \frac{a_{i}}{\Gamma\left(\alpha_{m}-\alpha_{i}\right)} \int_{t-\eta}^{t}(t-s)^{\alpha_{m}-\alpha_{i}-1} V(s) d s+\frac{1}{\Gamma\left(\alpha_{m}\right)} \int_{t-\eta}^{t}(t-s)^{\alpha_{m}-1} f(s, V(s)) d s\right)
$$$$
\leq 2 \varepsilon \text {. }
$$ 
Since by Lemma 3.2 the function $t \rightarrow v(t):=\beta(V(t))$ is continuous on $[0, t-\eta]$, it follows that $s \rightarrow(t-s)^{\alpha_{m}-1} g(V(s))$ is continuous on $[0, t-\eta]$. Hence, there exists $\delta>0$ such that

$$
\left\|(t-\tau)^{\alpha_{m}-1} g(V(\tau))-(t-s)^{\alpha_{m}-1} g(V(s))\right\|<\frac{\varepsilon}{2}
$$

and

$$
\|g(V(\xi))-g(V(\tau))\|<\frac{\varepsilon}{2 \eta^{\alpha_{m}-1}} .
$$

If $|\tau-s|<\delta$ and $|\tau-\xi|<\delta$ with $\tau, s, \xi \in[0, t-\eta]$, then it follows that

$$
\begin{aligned}
\left|(t-\tau)^{\alpha_{m}-1} g(V(\xi))-(t-s)^{\alpha_{m}-1} g(V(s))\right| \leq & \left|(t-\tau)^{\alpha_{m}-1} g(V(\tau))-(t-s)^{\alpha_{m}-1} g(V(s))\right| \\
& +(t-\tau)^{\alpha_{m}-1}|g(V(\xi))-g(V(\tau))| \\
< & \varepsilon
\end{aligned}
$$

that is,

$$
\left|(t-\tau)^{\alpha_{m}-1} g(V(\xi))-(t-s)^{\alpha_{m}-1} g(V(s))\right|<\varepsilon
$$

for all $\tau, s, \xi \in[0, t-\xi]$ with $|\tau-s|<\delta$ and $|\tau-\xi|<\delta$. Consider the following partition of the interval $[0, t-\eta]$ into $n$ parts $0=t_{0}<t_{1}<\cdots<t_{n}=t-\eta$ such that $t_{i-1}-t_{i}<\delta(i=$ $1,2, \ldots, n)$. By Lemma 3.2, for each $i$, there exists $s_{i} \in\left[t_{i-1}, t_{i}\right]$ such that $\beta\left(V\left(\left[t_{i-1}, t_{i}\right]\right)\right)=v\left(s_{i}\right)$, $i=1,2, \ldots, n$. Then we have (see [26], Theorem 2.2)

$$
\begin{aligned}
& \sum_{i=1}^{m-1} \frac{a_{i}}{\Gamma\left(\alpha_{m}-\alpha_{i}\right)} \int_{0}^{t-\eta}(t-s)^{\alpha_{m}-\alpha_{i}-1} V(s) d s+\frac{1}{\Gamma\left(\alpha_{m}\right)} \int_{0}^{t-\eta}(t-s)^{\alpha_{m}-1} f(s, V(s)) d s \\
& \subset \sum_{i=1}^{m-1} \frac{a_{i}}{\Gamma\left(\alpha_{m}-\alpha_{i}\right)} \int_{t-\eta}^{t}(t-s)^{\alpha_{m}-\alpha_{i}-1} V(s) d s+\sum_{j=1}^{n} \frac{1}{\Gamma\left(\alpha_{m}\right)} \int_{t_{i-1}}^{t_{i}}(t-s)^{\alpha_{m}-1} f(s, V(s)) d s \\
& \subset \sum_{i=1}^{m-1} \frac{a_{i}}{\Gamma\left(\alpha_{m}-\alpha_{i}\right)} \int_{t-\eta}^{t}(t-s)^{\alpha_{m}-\alpha_{i}-1} V(s) d s \\
& \quad+\frac{1}{\Gamma\left(\alpha_{m}\right)} \sum_{i=1}^{n}\left(t_{i}-t_{i-1}\right) \overline{\operatorname{conv}}\left\{(t-s)^{\alpha_{m}-1} f(s, u(s)) ; s \in\left[t_{i-1}, t_{i}\right], u \in V\right\},
\end{aligned}
$$

and so

$$
\begin{gathered}
\beta\left(\sum_{i=1}^{m-1} \frac{a_{i}}{\Gamma\left(\alpha_{m}-\alpha_{i}\right)} \int_{0}^{t-\eta}(t-s)^{\alpha_{m}-\alpha_{i}-1} V(s) d s+\frac{1}{\Gamma\left(\alpha_{m}\right)} \int_{0}^{t-\eta}(t-s)^{\alpha_{m}-1} f(s, V(s)) d s\right) \\
\leq \beta\left(\sum_{i=1}^{m-1} \frac{a_{i}}{\Gamma\left(\alpha_{m}-\alpha_{i}\right)} \int_{0}^{t-\eta}(t-s)^{\alpha_{m}-\alpha_{i}-1} V(s) d s\right) \\
\quad+\frac{1}{\Gamma\left(\alpha_{m}\right)} \sum_{i=1}^{n}\left(t_{i}-t_{i-1}\right) \beta\left(\overline{\operatorname{conv}}\left\{(t-s)^{\alpha_{m}-1} f(s, u(s)) ; s \in\left[t_{i-1}, t_{i}\right], u \in V\right\}\right)
\end{gathered}
$$




$$
\begin{aligned}
= & \beta\left(\sum_{i=1}^{m-1} \frac{a_{i}}{\Gamma\left(\alpha_{m}-\alpha_{i}\right)} \int_{0}^{t-\eta}(t-s)^{\alpha_{m}-\alpha_{i}-1} V(s) d s\right) \\
& +\frac{1}{\Gamma\left(\alpha_{m}\right)} \sum_{i=1}^{n}\left(t_{i}-t_{i-1}\right) \beta\left(\left\{(t-s)^{\alpha_{m}-1} f(s, u(s)) ; s \in\left[t_{i-1}, t_{i}\right], u \in V\right\}\right) \\
\leq & \beta\left(\sum_{i=1}^{m-1} \frac{a_{i}}{\Gamma\left(\alpha_{m}-\alpha_{i}\right)} \int_{0}^{t-\eta}(t-s)^{\alpha_{m}-\alpha_{i}-1} V(s) d s\right) \\
& +\frac{1}{\Gamma\left(\alpha_{m}\right)} \sum_{i=1}^{n}\left(t_{i}-t_{i-1}\right)\left(t-t_{i}\right)^{\alpha_{m}-1} \beta\left(f\left(\left[0, a_{0}\right] \times V\left[t_{i-1}, t_{i}\right]\right)\right) \\
\leq & \beta\left(\sum_{i=1}^{m-1} \frac{a_{i}}{\Gamma\left(\alpha_{m}-\alpha_{i}\right)} \int_{0}^{t-\eta}(t-s)^{\alpha_{m}-\alpha_{i}-1} V(s) d s\right) \\
& +\frac{1}{\Gamma\left(\alpha_{m}\right)} \sum_{i=1}^{n}\left(t_{i}-t_{i-1}\right)\left(t-t_{i}\right)^{\alpha_{m}-1} g\left(V\left[t_{i-1}, t_{i}\right]\right) \\
\leq & \beta\left(\sum_{i=1}^{m-1} \frac{a_{i}}{\Gamma\left(\alpha_{m}-\alpha_{i}\right)} \int_{0}^{t-\eta}(t-s)^{\alpha_{m}-\alpha_{i}-1} V(s) d s\right) \\
& +\frac{1}{\Gamma\left(\alpha_{m}\right)} \sum_{i=1}^{n}\left(t_{i}-t_{i-1}\right)\left(t-t_{i}\right)^{\alpha_{m}-1} g\left(V\left(s_{i}\right)\right) .
\end{aligned}
$$

Using (18) we have that

$$
\left|\left(t-t_{i}\right)^{\alpha_{m}-1} g\left(V\left(s_{i}\right)\right)-(t-s)^{\alpha_{m}-1} g(V(s))\right|<\varepsilon
$$

This implies that

$$
\begin{aligned}
& \frac{1}{\Gamma\left(\alpha_{m}\right)} \sum_{j=1}^{n}\left(t_{i}-t_{i-1}\right)\left(t-t_{i}\right)^{\alpha_{m}-1} g\left(V\left(s_{i}\right)\right) \\
& \quad \leq \frac{1}{\Gamma\left(\alpha_{m}\right)} \int_{0}^{t-\eta}(t-s)^{\alpha_{m}-1} g(V(s)) d s+\varepsilon(t-\eta) / \Gamma\left(\alpha_{m}\right) .
\end{aligned}
$$

By using (17) we claim that

$$
\beta\left(\sum_{i=1}^{m-1} \frac{a_{i}}{\Gamma\left(\alpha_{m}-\alpha_{i}\right)} \int_{t-\eta}^{t}(t-s)^{\alpha_{m}-\alpha_{i}-1} V(s) d s\right) \leq 2 \varepsilon .
$$

If we let

$$
\begin{aligned}
& A(t)=\frac{1}{\Gamma\left(\alpha_{m}\right)} \int_{t-\eta}^{t}(t-s)^{\alpha_{m}-1} f(s, V(s)) d s \\
& B(t)=\sum_{i=1}^{m-1} \frac{a_{i}}{\Gamma\left(\alpha_{m}-\alpha_{i}\right)} \int_{t-\eta}^{t}(t-s)^{\alpha_{m}-\alpha_{i}-1} V(s) d s,
\end{aligned}
$$


then $a+B(t) \subset A(t)+B(t)$ for $a \in A(t)$, which implies that $\beta(B(t)) \leq \beta(A(t)+B(t))<2 \varepsilon$. From relations (19) and (20), we obtain

$$
\begin{aligned}
& \beta\left(\sum_{i=1}^{m-1} \frac{a_{i}}{\Gamma\left(\alpha_{m}-\alpha_{i}\right)} \int_{0}^{t-\eta}(t-s)^{\alpha_{m}-\alpha_{i}-1} V(s) d s+\frac{1}{\Gamma\left(\alpha_{m}\right)} \int_{0}^{t-\eta}(t-s)^{\alpha_{m}-1} f(s, V(s)) d s\right) \\
& \quad \leq 2 \varepsilon+\frac{1}{\Gamma\left(\alpha_{m}\right)} \int_{0}^{t-\eta}(t-s)^{\alpha_{m}-1} g(V(s)) d s+\varepsilon(t-\eta) / \Gamma\left(\alpha_{m}\right) .
\end{aligned}
$$

Since

$$
\begin{aligned}
(Q V)(t) \subset & \sum_{i=1}^{m-1} \frac{a_{i}}{\Gamma\left(\alpha_{m}-\alpha_{i}\right)} \int_{0}^{t-\eta}(t-s)^{\alpha_{m}-\alpha_{i}-1} V(s) d s \\
& +\sum_{i=1}^{m-1} \frac{a_{i}}{\Gamma\left(\alpha_{m}-\alpha_{i}\right)} \int_{t-\eta}^{t}(t-s)^{\alpha_{m}-\alpha_{i}-1} V(s) d s \\
& +\frac{1}{\Gamma\left(\alpha_{m}\right)} \int_{0}^{t-\eta}(t-s)^{\alpha_{m}-1} f(s, V(s)) d s \\
& +\frac{1}{\Gamma\left(\alpha_{m}\right)} \int_{t-\eta}^{t}(t-s)^{\alpha_{m}-1} f(s, V(s)) d s
\end{aligned}
$$

then by virtue of (17) and (21), we have

$$
\begin{aligned}
\beta((Q V)(t)) & \leq \frac{1}{\Gamma\left(\alpha_{m}\right)} \int_{0}^{t-\eta}(t-s)^{\alpha_{m}-1} g(V(s)) d s+\varepsilon(t-\eta) / \Gamma\left(\alpha_{m}\right)+4 \varepsilon \\
& \leq \frac{1}{\Gamma\left(\alpha_{m}\right)} \int_{0}^{t}(t-s)^{\alpha_{m}-1} g(V(s)) d s+\varepsilon\left((t+4) / \Gamma\left(\alpha_{m}\right)\right) .
\end{aligned}
$$

As the last inequality is true for every $\varepsilon>0$, we infer

$$
\beta((Q V)(t)) \leq \frac{1}{\Gamma\left(\alpha_{m}\right)} \int_{0}^{t}(t-s)^{\alpha_{m}-1} g(V(s)) d s, \quad t \in\left[0, a_{0}\right]
$$

and thus by using (16) it follows that

$$
V(t) \leq \frac{1}{\Gamma\left(\alpha_{m}\right)} \int_{0}^{t}(t-s)^{\alpha_{m}-1} g(V(s)) d s \quad \text { for } t \in\left[0, a_{0}\right]
$$

Since $g(\cdot)$ is a Kamke function, then $V(t)=0$ for $t \in T_{0}$. Using Lemma 3.2, we infer

$$
\beta_{c}\left(V\left(T_{0}\right)\right)=\sup _{t \in T_{0}} \beta(V(t))=0
$$

Thus $V$ is relatively compact in $C\left(T_{0}, E_{w}\right)$. Therefore, taking a subsequence if necessary, we can assume that $\left\{u_{n}(\cdot)\right\}_{n \geq 1}$ converges weakly in $\widetilde{B}_{r}$ to a function $u(\cdot)$. Since $Q$ is weakly-weakly sequentially continuous, then $\left\{u_{n}-Q u_{n}\right\}_{n \geq 1}$ converges weakly to $u-Q u$ in $C\left(T_{0}, E_{w}\right)$. Recalling that the norm is weakly lower semicontinuous [27], we obtain that

$$
\lim _{n \rightarrow \infty} \sup \left\|u_{n}-Q u_{n}\right\|_{c} \geq\|u-Q u\|_{c}
$$


Then from (13) it follows that $\|u-Q u\|_{c}=0$, and so

$$
u(t)=u_{0}+\frac{1}{\Gamma\left(\alpha_{m}\right)} \int_{t-\eta}^{t}(t-s)^{\alpha_{m}-1} f(s, u(s)) d s, \quad t \in T_{0}
$$

Using Lemma 3.1, we conclude that $u(\cdot)$ is a solution of (9).

Remark 3.1 If $\alpha_{1}=\alpha_{2}=\cdots=\alpha_{m-1}=0$ and $\alpha_{m}=\alpha \in(0,1)$, then we obtain Theorem 5.1 from [21].

Let $E$ be a weakly sequentially complete space. It is known that if $f(\cdot, \cdot)$ is a continuous function from $T \times E_{w}$ into $E_{w}$, then the function $t \mapsto f(t, y(t))$ is Pettis integrable for every $A C$ function $y(\cdot): E \rightarrow E$ (see [28, Lemma 15]). Therefore, in the case of weakly sequentially complete spaces, we obtain the following result (see also [29]).

Corollary 3.1 Let $E$ be a weakly sequentially complete space. If $f(\cdot, \cdot): T \times E \rightarrow E$ is a continuous function from $T \times E_{w}$ into $E_{w}$ and conditions (h3)-(h4) in Theorem 3.1 hold, then (9) admits a solution $y(\cdot)$ on an interval $[0, a]$ with

$$
a_{0}=\min \left\{1,\left[\frac{r(1-a) \Gamma\left(\alpha_{m}+1\right)}{M}\right]^{1 / \alpha_{m}}\right\}
$$

Remark 3.2 If $\alpha_{1}=\alpha_{2}=\cdots=\alpha_{m-1}=0$ and $\alpha_{m}=1$, then we obtain some known results. In this case, Corollary 3.1 is a generalization of a result from [30] and [25]. Also, for any Banach space, the following result is a generalization of Theorem 2.1 in [31] (see also [13, $32-34]$ ) for $\alpha_{1}=\alpha_{2}=\cdots=\alpha_{m-1}=0$ and $\alpha_{m}=1$.

Corollary 3.2 If $f: T \times E \rightarrow E$ is a continuous function from $T \times E_{w}$ into $E_{w}$ such that $\mathrm{cl}_{w} f(T \times B) \in \mathcal{P}_{w k}(E)$, then (9) admits a solution on $[0, a]$ with

$$
a_{0}=\min \left\{1,\left[\frac{r(1-a) \Gamma\left(\alpha_{m}+1\right)}{M}\right]^{1 / \alpha_{m}}\right\}
$$

If $E$ is a reflexive Banach space, it is not necessary to assume any compactness conditions since in this case a subset of $E$ is weakly compact if and only if it is weakly closed and norm bounded. Thus, arguing similarly as in the proof of Theorem 3.1, we obtain the following result.

Corollary 3.3 [35] Let $E$ be a reflexive Banach space. If $: T \times E \rightarrow E$ is a continuous function from $T \times E_{w}$ into $E_{w}$ such that $\|f(t, y)\| \leq M$ for all $(t, y) \in T \times B_{r}$, then (9) admits a solution on $[0, a]$ with

$$
a_{0}=\min \left\{1,\left[\frac{r(1-a) \Gamma\left(\alpha_{m}+1\right)}{M}\right]^{1 / \alpha_{m}}\right\}
$$

Remark 3.3 If $\alpha_{1}=\alpha_{2}=\cdots=\alpha_{m-1}=0$ and $\alpha_{m}=1$, then we obtain Theorem 8 from [30], Theorem 3.1 from [25], and using the conditions of Corollary 3.3, we obtain some known results from [36]. 


\section{Competing interests}

All authors declare that they have no competing interests.

\section{Authors' contributions}

All authors carried out the proof. All authors conceived of the study, and participated in its design and coordination. All authors read and approved the final manuscript.

\section{Author details}

${ }^{1}$ Department of Mathematics, Texas A\&M University-Kingsville, Kingsville, TX, USA. ${ }^{2}$ Department of Mathematics, King Abdulaziz University, P.O. Box 80203, Jeddah, 21859, Saudi Arabia. ${ }^{3}$ Constantin Brancusi University, Republicii 1, Targu-Jiu, 210152, Romania. ${ }^{4}$ School of Mathematics, Statistics and Applied Mathematics, National University of Ireland, Galway, Ireland. ${ }^{5}$ Abdus Salam School of Mathematical Sciences, GC University, Lahore, Pakistan.

\section{Received: 7 August 2013 Accepted: 1 October 2013 Published: 08 Nov 2013}

\section{References}

1. Samko, S, Kilbas, A, Marichev, O: Fraction Integrals and Derivatives. Gordon \& Breach, New York (1993)

2. DicthIm, K: The Analysis of Fractional Differential Equations. Springer, Berlin (2010)

3. Babakhani, A, Daftardar-Gejji, V: Existence of positive solutions of nonlinear fractional differential equations. J. Math. Anal. Appl. 278, 434-442 (2003)

4. Daftardar-Gejji, V: Positive solutions of a system of non-autonomous fractional differential equations. J. Math. Anal. Appl. 302, 56-64 (2005)

5. Daftardar-Gejji, V, Jafari, H: Solving a multi-order fractional differential equation using adomian decomposition. Appl. Math. Comput. 189, 541-548 (2007)

6. Salem, HAH: Multi-term fractional differential equation in reflexive Banach space. Math. Comput. Model. 49, 829-834 (2009)

7. Salem, $\mathrm{HAH}$ : Monotonic solutions of multi-term fractional differential equations. Comment. Math. Prace Mat. 47(1), 1-7 (2007)

8. Salem, HAH: Global monotonic solutions of multi-term fractional differential equations. Appl. Math. Comput. 217 6597-6603 (2011)

9. Cichoń, M: Weak solutions of ordinary differential equations in Banach spaces. Discuss. Math., Differ. Incl. Control Optim. 15, 5-14 (1995)

10. Knight, WJ: Solutions of differential equations in Banach spaces. Duke Math. J. 41, 437-442 (1974)

11. Kubiaczyk, I, Szufla, S: Kneser's theorem for weak solutions of differential equations in Banach spaces. In: Lakshmikantham, V (ed.) Nonlinear Equations in Abstract Spaces, pp. 387-404 (1978)

12. Mitchell, AR, Smith, C: An existence theorem for weak solutions of differential equations in Banach spaces. In: Lakshmikantham, V (ed.) Nonlinear Equations in Abstract Spaces, pp. 387-404 (1978)

13. O'Regan, D: Fixed point theory for weakly sequentially continuous mapping. Math. Comput. Model. 27(5), 1-14 (1998)

14. Pettis, JP: On integration in vector spaces. Trans. Am. Math. Soc. 44, 277-304 (1938)

15. Knight, WJ: Absolute continuity of some vector functions and measures. Can. J. Math. 24(5), 737-746 (1972)

16. Schwabik, S, Guoju, Y: Topics in Banach Space Integration. World Scientific, Singapore (2005)

17. Kadets, WM: Non-differentiable indefinite Pettis integrals. Quaest. Math. 17(2), 137-139 (1994)

18. Munroe, ME: A note on weak differentiability of Pettis integrals. Bull. Am. Math. Soc. 52, 167-174 (1946)

19. Philips, RS: Integration in a convex linear topological space. Trans. Am. Math. Soc. 47, 114-145 (1940)

20. Arendt, W, Batty, C, Hieber, M, Neubrander, F: Vector-Valued Laplace Transforms and Cauchy Problems. Monogr. Math., vol. 96. Birkhäuser, Basel (2001)

21. Kubiaczyk, I, Szlufa, S: Kneser's theorem for weak solution of ordinary differential equations in Banach space. Publ. Inst. Math. 32(46), 99-103 (1982)

22. Salem, HAH, El-Sayed, SMA: A note of the fractional calculus in Banach spaces. Studia Sci. Math. Hung. 42(2), 115-130 (2005)

23. Dinuleanu, N: Vector Measures. Pergamon, New York (1967)

24. Singer, I: Linear functionals on the space of continuous mappings of a compact space into a Banach space. Rev. Roum. Math. Pures Appl. 2, 301-315 (1957)

25. Papageargiou, NS: Weak solutions of differential equations in Banach spaces. Bull. Aust. Math. Soc. 33, 407-418 (1986)

26. Geitz, RF: Geometry and the Pettis integral. Trans. Am. Math. Soc. 169(2), 535-548 (1982)

27. Edwards, RE: Functional Analysis. Holt, Rinehart \& Winston, New York (1965)

28. Cichoń, M: On solutions of differential equations in Banach spaces. Nonlinear Anal. 60, 651-667 (2005)

29. Dutkiewicz, A, Szufla, S: Kneser's theorem for weak solution of an integral equation with weakly singular kernel. Publ. Inst. Math. 77(91), 87-92 (2005)

30. Gomaa, AM: Weak and strong solutions for differential equations in Banach spaces. Chaos Solitons Fractals 18 687-692 (2003)

31. Kato, S: On existence of solutions of ordinary differential equations in Banach spaces. Funkc. Ekvacioj 19, 239-245 (1976)

32. Arino, O, Gautier, S, Penot, JP: A fixed point theorem for sequentially continuous mappings with application to ordinary differential equations. Funkc. Ekvacioj 27, 273-279 (1984)

33. Gautier, S, Penot, JP: Existence des courbes integrales pour les champs de vecteurs faiblement continus. Publications Mathematiques de Pau (1973)

34. O'Regan, D: Weak solutions of ordinary differential equations in Banach spaces. Appl. Math. Lett. 12, 101-105 (1999)

35. Salem, HAH, El-Sayed, AMA: Weak solution for fractional order integral equations in reflexive Banach space. Math. Slovaca 55(2), 169-181 (2005)

36. Szep, A: Existence theorem for weak solutions of differential equations in Banach spaces. Studia Sci. Math. Hung. 6, 197-203 (1971) 
10.1186/1687-1847-2013-302

Cite this article as: Agarwal et al.: Multi-term fractional differential equations in a nonreflexive Banach space. Advances in Difference Equations 2013, 2013:302

Submit your manuscript to a SpringerOpen ${ }^{\circ}$ journal and benefit from:

- Convenient online submission

- Rigorous peer review

- Immediate publication on acceptance

- Open access: articles freely available online

- High visibility within the field

- Retaining the copyright to your article

Submit your next manuscript at $\gg$ springeropen.com 